\title{
Towards the design of a new standard for composite stiffness identification
}

\author{
X. $\mathrm{Gu}^{1}, \mathrm{~F}$. Pierron ${ }^{2}$ \\ ${ }^{1}$ School of Aeronautics and Astronautics, Shanghai Jiao Tong University \\ Shanghai, China \\ Email: guxuesen@sjtu.edu.cn \\ ${ }^{2}$ Engineering and the Environment, University of Southampton, \\ Southampton, UK \\ Email: f.pierron@soton.ac.uk
}

\begin{abstract}
This paper presents a step towards the design of a novel test for simultaneous identification of all the stiffness components of orthotropic composite materials. A simulator was adopted to numerically simulate the whole identification process. Synthetic images were generated and then processed by Digital Image Correlation (DIC) to calculate the strain fields. The Virtual Fields Method (VFM) was used to identify the material stiffness parameters and error functions were finally defined to evaluate the identification error. Two steps of optimization were applied to obtain the best design variables of different specimens and the optimal DIC processing parameters. Four types of test configuration were simulated including short off-axis tensile test, short off-axis open-hole tensile test, off-axis Brazilian disc and offaxis unnotched Iosipescu test and the most promising configuration was identified.
\end{abstract}

Keywords: Digital image correlation, Virtual fields method, Simulated experiments, Test optimization

\section{Introduction and state of the art}

Composite materials are currently widely used in many sectors of industry thanks to their good performance to weight properties. The design of composite structures requires knowledge of the parameters driving their mechanical behaviour, among which their elastic stiffness components. In many cases, such as for unidirectional or cross-ply/woven laminates, their elastic behaviour can be assumed orthotropic and in a given plane (1-2), the stiffness behaviour depends on four independent parameters: $E_{11}$ and $E_{22}$, the Young's moduli in the two orthotropy directions, $v_{12}$, major Poisson's ratio and $G_{12}$, the shear modulus.

The identification of these stiffness components generally relies on the use of simple uniaxial tests for which there is an a priori knowledge of the stress field (statically determinate tests). For instance, $\mathrm{E}_{11}$ and $v_{12}$ can be obtained from a uniaxial tensile test along the fibre direction and $\mathrm{E}_{22}$ from a uniaxial tensile test perpendicular to the fibre direction, according to ASTM standard D3039 for instance [1]. For the shear modulus, several standard techniques are available, like the tensile test on a $[45 /-45]_{\mathrm{ns}}$ specimen 
(ASTM D3518, [2]), the double V-notch shear test (ASTM D5379, [3]), the rail shear test (ASTM D4255, [4]) or the off-axis tensile test [5], with oblique tabs for shallow angles [6]. These techniques are well established and based on robust deformation measurements from strain gauges or extensometers. However, they suffer from a number of shortcomings. First, they rely on stringent assumptions on geometry and boundary conditions to ensure the validity of the stress solution. This can cause spurious effects leading to biased stiffness identification [7]. Another issue is that the material has to be in a form that enables easy cutting of such test specimens. This is not so easy when testing in the through-thickness plane [8] for instance. Finally, this procedure is not very efficient as three tests are required to obtain four parameters. This leads to costly test campaigns and to the fact that spatial variations of properties are difficult to address because of the significant amount of test material that is required to perform the three tests.

With the development of full-field deformation measurements like Digital Image Correlation (DIC) for instance [9], new routes for composite stiffness identification have been proposed. The underpinning idea is to exploit the rich field information to conduct more complex tests and use an inverse identification tool to determine the required parameters. Research efforts towards this goal started in the late eighties [10] for plate bending but the bulk of the work targeted at in-plane loading tests dates back to the early 2000. Different types of full-field measurements have been used for this purpose: DIC [11], speckle interferometry [12], moiré interferometry [13] and grid method [14], among the most popular. Displacement or strain fields are then processed using an inverse identification technique to extract the stiffness parameters from the measured field data. Several techniques can be used for this purpose as reported in [15]. Finite element model updating (FEMU) consists in building up a finite element model of the test and constructing a cost function as the difference between measured and computed quantities [11, 13]. An alternative is the Virtual Fields Method (VFM) which uses the measured strain field to directly extract the stiffness parameters from a specific use of the principle of virtual work [14, 16]. Both techniques are nominally equivalent in elasticity, as shown in [17], the VFM being more computationally efficient as no iterative finite element computations are required.

One of the difficulties in using the approach described above is the choice of the test configuration. Sometimes, the test geometry is conditioned by the manufacturing process of the test piece $[11,18]$ but for generic testing of thin plates, the design space for both test geometry and loading is quite wide. Sometimes, the test configurations used with the above methodology have been recycled from existing tests like the open-hole tensile test [13], the Arcan test [19], or slightly adapted as for the unnotched 
Iosipescu test $[12,20]$. There have been attempts to design specific tests, for instance a T-shaped specimen loaded in tension/bending [21] but only recently have research efforts been targeted at more systematic specimen optimization. Muhammad et al. [22] attempted to optimize the load configuration of a rectangular composite plate in bending and validated the results experimentally. Their approach was based on the use of the sensitivity to noise coefficients provided by the optimized VFM [23]. The same procedure has been used for in-plane orthotropic stiffness components in the unnotched Iosipescu test [12] and the modified Arcan test [19].

If such procedures are ever going to be used as new standard tests to replace existing ones, it is paramount that the uncertainty of the identified parameters be realistically evaluated. For this, the simple model of uncorrelated strain noise on which the test optimizations cited above have been performed is not sufficiently realistic. First, strain noise is correlated since all components derive from the measured displacement components and even these are not uncorrelated as they derive from the same subset in DIC, for instance. In reality, the main source of noise is the variation of grey level at each pixel caused mainly by the sensor of the camera. This was considered in [11] in a DIC/FEMU procedure to produce random error maps. However, the random error generated by camera noise is not the only one. A systematic error is also produced from the limited spatial resolution of full-field measurements. Indeed, the data are collected at a certain number of discrete points (subset of pixels in DIC, lines for the grid method) and therefore, high strain gradients are likely to be underestimated by such measurements, all the more since in the elastic regime, extra regularization needs to be included to make the identification noise-robust. This is illustrated in Fig. 16 in [24] for instance.

Because of the complexity of the combined measurement and identification chain, such uncertainty quantification can only be addressed by numerical simulations. Such a simulator was first proposed by Rossi and Pierron [25] using the grid method and recently extended to DIC [24]. Its application to orthotropic PVC foam stiffness identification has enabled both test configuration optimization and realistic confidence interval predictions [26].

The objective of the present paper is to use this simulator to approach in a rational way the design of a potential new test standard to identify the complete set of orthotropic stiffness components in a plane from a single test. Several configurations are explored and related to test parameters, like dimensions or orthotropy axis direction. A sum of the systematic and random errors is calculated as the total identification error. The error arising from the load measurement has been neglected here as it is generally much lower than that arising from the imaged-based strain measurements when the load cell is of 
appropriate capacity compared to the measured load. Other sources of error such as camera misalignment, lens distortion, non-uniform lighting are not considered in this paper either and will be included in further studies. Detailed simulations are presented in this article to reach a conclusion about the suitability of each of the explored test configurations. Confidence intervals on each stiffness component are also provided. Finally, considerations like ease of specimen preparation or robustness of loading conditions are added to make the final choice of the most promising candidate. This will be the base of future experimental validation before such a test can be proposed to the testing community.

\section{Simulation of the experiments}

In order to compare different experimental configurations, the whole identification chain has been numerically simulated following the procedures reported in [24, 25]. The flow chart of the simulator is shown in Figure 1.

Figure 1 - Flow chart of the identification simulator

Firstly, a finite element model is built up to simulate the displacement fields of a loaded specimen, with the input of material properties, geometry and boundary conditions of the real test. Then, the deformed image is obtained by imposing the displacement fields calculated by finite element on the reference image. This uses a subsampling to reduce the interpolation errors, details can be found in [24]. Grey level white noise can be added to both generated images to simulate a realistic image capture. Digital Image Correlation (DIC) is then applied to calculate the strain fields, in the same way as in the real experimental procedure. With the strain fields and applied force, stiffness parameters can finally be identified using the VFM. The identification error can be calculated by comparing the identified stiffness parameters with the reference values used as input in the finite element model. The following provides information about the different steps in this procedure.

\subsection{Test configurations}

The objective of this article is to compare a few potential tests for robust and accurate simultaneous identification of orthotropic stiffness components, with a view to defining a new standard. As a consequence, this ideal test candidate should not only lead to accurate results but should also involve a specimen which is relatively easy to manufacture and to load. The design space is nearly infinite and to start exploring it rationally, the first attempt presented here focuses on a few configurations arising from the second author's past experience. The other criterion used to refine the design space is that the design 
variables should be limited so as not to render the optimization procedure too computationally intensive at this stage. This will be explored in future work.

Four types of test configuration have been considered here, including the short off-axis tensile test (SOAT), short off-axis open-hole tensile test (SOAHT), off-axis Brazilian disc (OABD) and off-axis unnotched Iosipescu test (OAUIT). The complete sets of design variables of each test are reported in Table 1. The FE models of the four types of test are shown in Figure 2.

\begin{tabular}{l|c}
\multicolumn{2}{c}{ Design variable(s) } \\
\hline SOAT & $H(25 \mathrm{~mm}-60 \mathrm{~mm}$, by increments of $5 \mathrm{~mm})$ \\
SOAHT & $\theta\left(10^{\circ}-80^{\circ}\right.$ by increments of $\left.10^{\circ}\right)$ \\
& $D(4 \mathrm{~mm}-16 \mathrm{~mm}$ by increments of $2 \mathrm{~mm})$ \\
OABD & $\theta\left(10^{\circ}-80^{\circ}\right.$ by increments of $\left.10^{\circ}\right)$ \\
OAUIT & $\theta\left(10^{\circ}-80^{\circ}\right.$ by increments of $\left.10^{\circ}\right)$ \\
& $L(10 \mathrm{~mm}-50 \mathrm{~mm}$, by increments of $10 \mathrm{~mm})$ \\
\hline
\end{tabular}

Table 1 - Design variables and the value ranges for all the simulated tests

Figure 2 - FE models of the four types of test: (a) Short off-axis tensile test (SOAT); (b) Short offaxis open-hole tensile test (SOAHT); (c) Off-axis Brazilian disc (OABD); (d) Off-axis unnotched Iosipescu test (OAUIT)

The first test is inspired from a recent study on orthotropic PVC foams [19, 26] where it was found that a short specimen loaded in off-axis tension was the most appropriate over other configurations involving tension/shear and different off-axis angles. This has led to the so-called 'short off-axis tensile' test, or SOAT. The idea here is to use the shear-tension coupling to an advantage to create a fully heterogeneous stress state. The design variables are the aspect ratio $L / H$ and the off-axis angle (see Figure 2(a)). The extreme angles $0^{\circ}$ and $90^{\circ}$ were omitted as for certain configurations like the SOAT or the OABD, they would not allow complete identification of the stiffness components. As for the others, they would not provide interesting configurations as already shown in [24, 25] for the OAUIT.

The second test (SOAHT) combines the SOAT geometry with features from open-hole tensile tests used in [13]. The heterogeneity arises both from the shear-tension coupling and the presence of the hole, which should give more flexibility than the first test provides. To keep the problem simple, only the offaxis angle and the hole diameter are considered as design variable (see Figure 2(b)). The aspect ratio $L / H$ is set to 1.2 which is the aspect ratio of the CCD sensor of the camera considered in the present 
simulations as it has been found previously $[12,24,25]$ that this was a strong constraint. The result from the first test should confirm this finding.

The third test (OABD) considered here is the off-axis Brazilian disc test [27]. This test is mainly used to study the tensile fracture of brittle materials but has also been used in experimental mechanics as a benchmark as there is an analytical solution to this problem for isotropic materials. It produces a naturally heterogeneous stress state, which will be enhanced here by the adjustment of the off-axis angle, which is the sole design variable for this test (see Figure 2(c)).

The final test considered here is the off-axis unnotched Iosipescu test (OAUIT). This has been the object of many studies in the past $[12,20,24,25,28,29]$. This will serve as a reference to compare the other tests against. The two design variables are the free length $L$ and the off-axis angle $\theta$, see Figure 2(d). The heterogeneity arises from the combined bending and shear stresses in the active central part of the specimen (controlled by $L$ ), enhanced by the possibility to adjust the off-axis angle.

Table 2 shows the a priori advantages and drawbacks of the selected configurations. The next section presents the finite element models developed for these four tests.

\begin{tabular}{|c|c|c|}
\hline Test type & Advantages & Drawbacks \\
\hline $\begin{array}{l}\text { Short off-axis } \\
\text { tensile }\end{array}$ & $\begin{array}{l}\text { - Specimen easy to prepare } \\
\text { - Use of standard test machine }\end{array}$ & $\begin{array}{l}\text { - Requires excellent gripping to ensure } \\
\text { heterogeneity }\end{array}$ \\
\hline $\begin{array}{l}\text { Short open-hole } \\
\text { off-axis tensile }\end{array}$ & $\begin{array}{l}\text { - Specimen relatively easy to } \\
\text { prepare } \\
\text { - Use of standard test machine }\end{array}$ & $\begin{array}{l}\text { - Needs a hole (potential drilling damage } \\
\text { which may cause premature softening } \\
\text { at the hole) }\end{array}$ \\
\hline $\begin{array}{l}\text { Off-axis Brazilian } \\
\text { disc }\end{array}$ & $\begin{array}{l}\text { - Easy to load (compressive } \\
\text { platens) }\end{array}$ & $\begin{array}{l}\text { - Specimen difficult to prepare (water jet } \\
\text { cutting), defects may cause premature } \\
\text { crushing under compression } \\
\text { - Difficulty to ensure 2D loading }\end{array}$ \\
\hline $\begin{array}{l}\text { Unnotched } \\
\text { Iosipecu }\end{array}$ & - Specimen easy to prepare & $\begin{array}{l}\text { - Fixture elaborate and expensive [30] } \\
\text { - Issues with through-thickness strain } \\
\text { distributions [7] }\end{array}$ \\
\hline
\end{tabular}

Table 2 - Advantages and drawbacks of the different test configurations considered here

\subsection{Finite element (FE) models and normalization}

The four tests were simulated in this paper. All the FE models were built up using 2D quadratic elements (CPS4) with Abaqus version 6.10. In order to ensure that convergent FE models were used for the simulations, a convergence study of the mesh size was performed by comparing the average identification error of all the stiffness parameters. A mesh size of $0.2 \mathrm{~mm}$ was chosen and fixed for all the simulations. As illustrated in Figure 2, the specimen coordinate system is (X, Y) and the material coordinate system is $(1,2)$, with 1 as the direction of the fibre (larger stiffness). In order to compare the 
different test configurations under various loading conditions, a normalization procedure was performed during the computation of the displacement field. Since the models are linear elastic, any magnitude of load will result in scaled up or scaled down displacements. However, the random part of the identification error caused by camera noise is sensitive to the level of displacements (signal to noise ratio depends on the signal magnitude for a given noise magnitude). In other words, one should perform the identification with the maximum possible load applied to the test specimen to maximize the signal to noise ratio. Therefore, comparing the results for say the same load magnitude will lead to biased results as the specimens have different overall stiffnesses. In practice however, this is limited by the domain of linear elastic behaviour of the material. In order to be able to realistically compare the different test configurations, a deformation normalization procedure has been introduced as described in [24, 25]. For each test, the loading force is scaled up or down so that the stress levels in the specimens do not go above certain stress thresholds. In the fibre direction, $\sigma_{11}$ should be less than the related strengths in tension, $S_{+1}$ or compression, $\mathrm{S}_{-1}$. Similarly, in the transverse direction, $\sigma_{22}$ should be less than $S_{+2}$ in tension and $S_{-2}$ in compression. In shear, because of the non-linear behaviour, a linearity threshold is used to limit the magnitude of $\sigma_{12}$ (which is denoted $\sigma_{6}$ in following sections). The values used in this study are typical of a carbon/epoxy unidirectional and provided in Table 3. In practice, the limiting factors are always $S_{-2}$ (transverse tension) and $S_{6}$ (shear).

\begin{tabular}{lc|cc}
\hline \multirow{2}{*}{ Reference stiffness (GPa) } & \multicolumn{2}{|c}{ Maximum stress (MPa) } \\
\hline$Q_{11}$ & 125.30 & $S_{+1}$ & 1500 \\
$Q_{22}$ & 8.26 & $S_{-1}$ & 1200 \\
$Q_{12}$ & 2.64 & $S_{+2}$ & 50 \\
$Q_{66}$ & 4.00 & $S_{-2}$ & 250 \\
& $Q_{11}=\frac{S_{6}}{1-v_{12}^{2} * E_{22} / E_{11}}$ & \\
& $Q_{22}=\frac{E_{22}}{1-v_{12}^{2} * E_{22} / E_{11}}$ \\
$Q_{12}$ & $=\frac{E_{22} * v_{12}}{1-v_{12}^{2} * E_{22} / E_{11}}$ \\
$Q_{66}$ & $=G_{12}$
\end{tabular}

Table 3 - Reference stiffness components and maximum stress values (from [25]), together with the relationships between the stiffness parameters $\left(Q_{i j}\right)$ and the engineering constant $\left(E_{11}, E_{22}, G_{12}\right.$ and $\left.v_{12}\right)$. 
It is worth noticing that in the simulation of the OABD specimen, because of the point contact between the specimen and the indenter, serious stress concentrations occur in that area. Applying the scaling on the maximal stress would strongly penalize this test even though in practice, the load would be spread over a certain contact area and the real stress concentration would be much less critical. In an attempt to address this issue, two rows of elements at both the top and bottom contact points of the specimen were deleted to mitigate this effect. It should be pointed out that this is done only for the normalization process, the identification does take all the data into account.

\subsection{Image deformation and Digital Image Correlation (DIC)}

The image deformation procedure is the same as that described in [24] and is a refinement of a previous version initially developed for large strains [31]. The refinement concerns a subsampling procedure that mitigates interpolation errors. This has been evaluated in detail in [24] and will not be recalled here.

The reference image is generated by a National Physical Laboratory (NPL) synthetic image generator by simulating white spots sprayed over a uniform black background (see Figure 3). The resolution of the reference image is $2400 \times 2000$ pixel $^{2}$, the same as the CCD size of the simulated camera. The simulated speckle size is 4 pixels with 8 bit depth. The grey histogram of the reference image is given in Figure 4 .

Figure 3 - Reference speckle pattern, with a detail

Figure 4 - Grey histogram of the reference image

To evaluate the random part of the error, noise has been added to the grey level images. The noise model used here is Gaussian white noise with a standard deviation of $1 \%$ of the dynamic range of the camera, hence 2.56 grey levels for the 8-bit CCD camera considered here. This is based on the authors’ experience over a range of uncooled cameras, where noise typically ranges between 0.5 and $1 \%$ of the dynamic range, regardless of the latter as noise seems to scale up with bit-depth. The exact value of the noise in that range is of little consequence, as illustrated in Fig. 22 in [24]. It should be noted that real noise somewhat departs from the ideal zero mean Gaussian distribution as shown for instance in [32]. However, this has proved to have only a small influence on the error [33] and the Gaussian distribution was used for the sake of convenience. 
2D Digital Image Correlation is now a relatively mature technique and it was considered unnecessary to recall its principle here, all details can be found in [9]. The MatchID package was used here (www.matchidmbc.com). The required information will be provided along with the results from the simulations (see Table 5).

\subsection{The Virtual Fields Method (VFM)}

The VFM is an inverse technique using full-field measurements to identify constitutive material parameters. Its main feature is that, compared to Finite Element Model Updating (FEMU, see [15]), it does not require iterative forward computations and is therefore very computationally efficient. It is beyond the scope of the paper to recall the VFM theory for anisotropic linear elasticity which is now well established. The interested reader can find all the details in [16]. Here, piecewise optimized virtual fields have been used as in [19]. The size of the virtual mesh which defines the virtual displacement field in the reference coordinate system is 4 by 4 elements (total of 16 virtual elements). The boundary conditions for all test configurations are listed in Table 4. The distribution of force along the loading boundary is unknown, only the resultant vertical load is known as it is measured by the load cell of the test machine. Therefore, by selecting a virtual displacement field that has a constant virtual vertical displacement along this boundary (see Table 4), the unknown distribution is filtered out and only the measured resultant appears in the final equation. Also, by zeroing the horizontal component of the virtual displacement along the same boundary, the unknown horizontal reaction forces arising from the specimen gripping are cancelled out from the final equations. This illustrates the filtering capabilities of the principle of virtual work. In this case, the virtual fields are not kinematically admissible but VFM-admissible (ie, no unknown remains in the VFM equations).

\begin{tabular}{|c|c|c|c|c|}
\hline & SOAT & SOAHT & OABD & OAUIT \\
\hline Virtual mesh size & \multicolumn{4}{|c|}{$4 \times 4$ elements } \\
\hline Boundary conditions & \multicolumn{3}{|c|}{$\begin{array}{l}\text { At the bottom boundary: } \\
\mathrm{u}_{\mathrm{X}}^{*}=\mathrm{u}_{\mathrm{Y}}^{*}=0 \text {. } \\
\text { At the top boundary: } \\
\mathrm{u}_{\mathrm{X}}^{*}=0, \mathrm{u}_{\mathrm{Y}}^{*}=\text { constant. }\end{array}$} & $\begin{array}{l}\text { At the left boundary: } \\
\mathrm{u}_{\mathrm{X}}^{*}=\mathrm{u}_{\mathrm{Y}}^{*}=0 . \\
\text { At the right boundary: } \\
\mathrm{u}_{\mathrm{X}}^{*}=0, \mathrm{u}_{\mathrm{Y}}^{*}=\text { constant. }\end{array}$ \\
\hline
\end{tabular}

Table 4 - Virtual mesh size and boundary conditions for all test configurations, $\mathrm{u}_{\mathrm{X}}^{*}$ and $\mathrm{u}_{\mathrm{Y}}^{*}$ are the virtual displacements along the horizontal and vertical directions, respectively

\subsection{Error functions}

To compare the different configurations, error functions are introduced as in [34]. 


$$
C_{1}=0.25 \sum_{i j}\left(\left|\frac{Q_{i j}^{i d}-Q_{i j}^{r e f}}{Q_{i j}^{r e f}}\right|\right), i j=[11,22,12,66]
$$

where $Q_{i j}^{i d}$ are the identified stiffness parameters, and $Q_{i j}^{r e f}$ are the reference values. $i j$ represents the indices of the non-zero elements in the stiffness matrix of the orthotropic material. This represents the systematic error (or bias) when no noise is introduced.

The random error was simulated by adding grey level white noise to the synthetic images. Error function $C_{2}$ was defined as the average value of the ratios of the standard deviations to the reference stiffness parameters (coefficients of variation).

$$
C_{2}=0.25 \sum_{i j}\left(\frac{\sigma_{i j}^{i d}}{Q_{i j}^{r e f}}\right), i j=[11,22,12,66]
$$

where $Q_{i j}^{r e f}$ are the reference values, and $\sigma_{i j}^{i d}$ the standard deviations of the identified parameters over 30 repetitions.

The total error function $C_{3}$ includes both the systematic and random errors as:

$$
C_{3}=0.25 \sum_{i j}\left(\left|\frac{Q_{i j}^{i d}-Q_{i j}^{r e f}}{Q_{i j}^{r e f}}\right|+\frac{2 \sigma_{i j}^{i d}}{Q_{i j}^{r e f}}\right), i j=[11,22,12,66]
$$

This corresponds to the maximal error within a 95\% confidence interval.

\section{Optimization of the test configuration}

Ideally, the optimization should address all parameters from the identification chain: the test configuration (see design variables in Table 1), the DIC and VFM parameters (subset and step sizes, number of virtual elements etc.). However, this is a very complex non-linear optimization problem with many parameters and as it has been performed in [26], the problem will be decomposed in two steps. First, the test configurations will be optimized while keeping DIC and VFM parameters fixed. Then, for each optimal configuration of each test type, DIC and VFM parameters will be optimized. This will then lead to the ranking of the different test types, from the most to the less promising, which is the objective of the present paper. In the future, the full optimization problem will be tackled and the present results will serve as benchmark to understand this complex optimization.

\subsection{Optimization of the design variables of the simulated experiments}

\subsubsection{Systematic error for the SOAT specimen}

The systematic error is first considered. For this, no noise has been added to the synthetic images. By using the simulator mentioned in the previous section, the reference and deformed images were processed by the DIC software to calculate the displacement fields. The DIC parameters were kept constant during the processing and listed in Table 5. 


\begin{tabular}{c|c} 
Subset size & 31 \\
Step size & 15 \\
Shape function & Affine \\
Interpolation function & Bicubic spline interpolation \\
Correlation criterion & Approximated normalized sum of squared \\
difference \\
Pre-smoothing & Gaussian kernel size 5 \\
Strain calculation & Least-square fit of a bilinear function over \\
the $\mathrm{N}$ by N strain window & $\mathrm{N}=5$ \\
Strain window size & $2400 \times 2000$ pixel $^{2}$ \\
Camera resolution & $4 \times 4$ pixel $^{2}$ \\
\hline Speckle size &
\end{tabular}

Table 5 - DIC processing parameters using in the first step of the optimization

The $C_{1}$ error function for SOAT is plotted as a contour map in Figure 5. The two axes of the graphs represent the two design variables (specimen height $H$ and off-axis angle $\theta$ ), respectively (the length of the specimen $L$ was fixed at $48 \mathrm{~mm}$ ). As can be seen on this plot, some configurations lead to relatively large errors when the off-axis angle is close to $0^{\circ}$ or $90^{\circ}$. Interestingly, the optimal specimen height is $40 \mathrm{~mm}$; this corresponds to the aspect ratio of the camera used for the simulation $(48 / 40=2400 / 2000)$. This was also the conclusion from [24, 25], it corresponds to the best use of the spatial resolution of the camera. As for the angle, there is a rather wide range of values leading to low systematic errors, between $20^{\circ}$ and $70^{\circ}$.

Figure 5 - $C_{1}$ error function for SOAT (systematic error)

It is also interesting to look at the contribution of each stiffness component to the error. Figure 6 illustrates the error contribution of each identified stiffness parameter $\left(Q_{11}, Q_{22}, Q_{12}\right.$ and $\left.Q_{66}\right)$ separately. It is clear that the identification errors of parameters $Q_{11}, Q_{22}$ and $Q_{66}$ mainly depends on the off-axis angle of the specimen. As expected, shallow angles lead to better identification of $Q_{22}$ and larger angles, of $Q_{11}$ (with the convention in Figure 2 where small angles refer to horizontal fibre orientation). For the shear modulus, larger angles are better as shallow angles lead to concentrated strains near the grip which cause high spatial resolution related bias. As for $Q_{12}$, it contributes the most to the systematic error as expected and therefore, its error map strongly influences that of the total error. This parameter is only well identified if both $Q_{11}$ and $Q_{22}$ are accurately known, which leads to a compromise for the off-axis angle of about $30^{\circ}$, which can also be seen on the total error map. 
Figure 6 - Systematic error contribution to $C_{1}$ of each identified stiffness parameter for the SOAT configuration

\subsubsection{Random error for the SOAT specimen}

To evaluate the random error, 30 repeats of simulations with different copies of the grey level white noise were run. Error function $C_{2}$ is considered here and the results are shown on Figure 7. It is clear that again, the off-axis angle has the largest influence and the patterns are very similar to that of Figure 6 . The main difference concerns $Q_{12}$ for which the minimum has shifted to larger angles. This is a consequence of the normalization procedure described earlier as the random error is highly influenced by the actual strain levels in the specimen which depend on the maximum applied load which in turn depends on the relative stress to strength values.

Figure 7 - Coefficients of variation (error function $C_{2}$ ) of each identified stiffness parameter for the

$$
\text { SOAT configuration }
$$

By combining the systematic and random errors together in error function $C_{3}$, an estimate of the minimal identification error can be reached and shown in Figure 8.

Figure 8 - Total error function $C_{3}$ for the SOAT configuration

According to Figure 8, optimal identification is obtained when the height of the specimen is close to $40 \mathrm{~mm}$ and the off-axis angle is about $40^{\circ}$. The optimal configuration leads to an average error of about $3.5 \%$, three times lower than a bad configuration, like $\left(25 \mathrm{~mm}, 10^{\circ}\right)$. But this average error hides large differences between stiffness components, as shown in Figure 9. Since $Q_{12}$ has both the largest systematic and random errors, the overall optimal configuration exists in the area around the best identification of $Q_{12}$, which comes from a compromise between $Q_{11}$ and $Q_{22}$ with moderate off-axis angles. As for $Q_{66}$, the total error is also relatively low in the optimal identification area of $Q_{12}$.

Figure 9 - Total error function $C_{3}$ of each identified stiffness parameter for the SOAT configuration

A comparison of strain components between the optimal test configuration $\left(40 \mathrm{~mm}, 40^{\circ}\right)$ and a bad one ( $30 \mathrm{~mm}, 80^{\circ}$ ) is given in Figure 10 . For the bad configuration, the $\varepsilon_{22}$ component is very small compared to the others and the shear strain is low over a large part of the specimen. For the optimal configuration, the strain components are more balanced and the gradients less steep. 
Figure 10 - Simulated DIC strain maps (with noise) for (a) the optimal SOAT configuration (40 mm, $40^{\circ}$ ) and (b) a bad one $\left(30 \mathrm{~mm}, 80^{\circ}\right)$

Figure 11 shows the strain fields for the optimal SOAT configuration obtained from the FE model and simulated DIC without and with noise. During actual measurements, the low-pass spatial filtering effect of DIC smooths out the high gradients of strain and the random noise covers the whole field of view. By considering these effects, the simulated DIC with noise generates more realistic strain fields than the FE simulation and simulated DIC without noise.

Figure 11 - Strain fields for the optimal SOAT configuration (40 mm, 40), obtained from the FE model, the simulated DIC without and with noise.

\subsubsection{SOAHT specimen}

The same routine was applied to the SOAHT specimen with the two design variables for this configuration: the diameter $D$ of the open hole and the off-axis angle $\theta$. The length $L$ and the height $H$ of the specimen were fixed at $48 \mathrm{~mm}$ and $40 \mathrm{~mm}$ respectively, to reproduce the aspect ratio of the camera simulated here (see comments in the previous section and in [24, 25]). All error function plots are provided in Figure 12. The best identification is obtained for $D=10 \mathrm{~mm}$ and $\theta=40^{\circ}$. For the individual parameters, the results are very similar to that of the SOAT specimen for the off-axis angle.

Figure 12 - (a) $C_{1}$ (bias), (b) $C_{2}$ (random) and (c) $C_{3}$ (total) error funtions for the SOAHT configuration

\subsubsection{OABD specimen}

The OABD has only one design variable, the off-axis angle $\theta$. The diameter $D$ of the disk was fixed at $100 \mathrm{~mm}$ and will only depend on the camera chip size and lens magnification in a practical experiment. The results are plotted in Figure 13. In both $C_{1}$ and $C_{2}, Q_{66}$ is the parameter with the lowest systematic and random erros, while $Q_{12}$ has the largest. As expected, the best identifications of $Q_{11}$ and $Q_{22}$ were obtained when the related direction was aligned with the loading. Figure 13(c) shows that the optimal identification occurs when $\theta$ is $40^{\circ}$.

Figure 13 - (a) Individual $C_{1}$ (bias), (b) Individual $C_{2}$ (random) and global $C_{3}$ (total) error funtions for the OABD configuration 


\subsubsection{OAUIT specimen}

The same routine applied to the SOAT and SOAHT configurations was used for the OAUIT specimen with the two design variables: free length $L$ and the off-axis angle $\theta$. The height of the specimen was fixed at $20 \mathrm{~mm}$. All error functions are plotted in Figure 14. It demonstrates that the total error worsens when the off-axis angle exceeds $60^{\circ}$. Also the aspect ratio of the specimen plays an important role as it did in the SOAT configuration. The best identification is obtained for $L=30 \mathrm{~mm}$ and $\theta=50^{\circ}$.

Figure 14 - (a) $C_{1}$ (bias), (b) $C_{2}$ (random) and $C_{3}$ (total) error funtions for the OAUIT configuration

\subsubsection{Comparison between the test configurations after the first stage}

After the first step, the optimal design variables for each test configuration were recorded and listed in Table 6 with the corresponding errors. It is interesting to note the difference between the SOAT and SOAHT configurations. The first one has a low systematic error but a large random error whereas it is the other way around for the second one. The presence of the hole increases the systematic error because of the strain gradients near the hole, but the strain contents is richer leading to lower random errors. The OABD has the largest total error which is not surprising, since strains mainly concentrate near the contact points at both top and bottom of the disc. As to the OAUIT, both the systematic and random errors are relatively low. This confirms that this test is a good candidate as this was validated experimentally in the past [12,20,36,37], however, the fixture is expensive and prone to through-thickness strain heterogeneities [7].

This illustrates the importance of the image deformation simulation in the test design procedure in understanding the benefits of each test configuration.

\begin{tabular}{c|cccc}
\hline & SOAT & SOAHT & OABD & OAUIT \\
\hline $\begin{array}{c}\text { Optimal values } \\
\text { of the design } \\
\text { variables }\end{array}$ & $\begin{array}{c}L=40 \mathrm{~mm} \\
\theta=40^{\circ}\end{array}$ & $\begin{array}{c}D=10 \mathrm{~mm} \\
\theta=40^{\circ}\end{array}$ & $\theta=40^{\circ}$ & $\begin{array}{c}L=30 \mathrm{~mm} \\
\theta=40^{\circ}\end{array}$ \\
\hline$C_{1}$ & 0.0055 & 0.0136 & 0.0155 & 0.0085 \\
$C_{2}$ & 0.0142 & 0.0059 & 0.0166 & 0.0083 \\
$C_{3}$ & 0.0339 & 0.0254 & 0.0487 & 0.0251 \\
\hline
\end{tabular}

Table 6 - Optimal values of the design variables and the corresponding errors of the four simulated tests 
Now that optimal configurations have been identified with a particular set of DIC parameters, it is important to optimize these parameters to check if the total error can be reduced further.

In this section, the design variables studied in the previous section are fixed at the optimal values. The DIC subset size and strain window size (smoothing parameter) are considered as the new design variables for each test. The subset size was varied from 21 to 51 with an increment of 10, and the strain window size was varied from 3 to 25 by increments of 2 . The step size was fixed at $50 \%$ of the subset size. All other DIC parameters from Table 5 are kept the same.

The systematic and random errors (twice the coefficient of variation) for the SOAT specimen are plotted in Figure 15. The systematic error increases with strain window size, as expected as larger strain windows reduce local strain gradients more. It also increases with subset size, for the same reason. The random error behaves in an opposite way.

Figure $15-C_{1}$ and twice $C_{2}$ for the SOAT specimen, with different subset and strain window sizes

The error decreases with strain window and subset size. When both contributions are added together (Figure 16(a)), a minimum can be identified for each subset size, and then between strain window sizes. The best set of parameters is a subset size of 41 with a strain window of 9 data points.

Figure $16-C_{3}$ for (a) SOAT; (b) SOAHT; (c) OABD; (d) OAUIT, with different subset and strain window sizes

The same procedure was applied to the SOAHT, OABD and OAUIT specimens. Figure 16 (b-d) shows the total error for the three tests. The general trend is similar to that of the SOAT specimen.

The optimal combinations of the two design variables for each test configuration are listed in Table 7 together with the total error before and after DIC parameter optimization. It shows that the SOAHT gives the best overall identification result when the subset is 41 pixels and the strain window size is 11 data points. The OAUIT specimen provides a close enough value to that of the SOAHT one and could be a suitable candidate too. However, the complexity of its loading fixture and the potential through-thickness strain heterogeneities (as reported in [7]) somewhat disqualifies it. The SOAT comes next but since drilling a hole in a composite specimen does not really constitute a problem, it is thought that the SOAHT specimen is more appropriate. Finally, the OABD has the largest identification error among all four tests and is discarded. This is not so surprising since the strains are concentrated at the contact points and suffer both from a large systematic error and a smaller load allowance because of local stress concentrations. 


\begin{tabular}{ccccc}
\hline & SOAT & SOAHT & OABD & OAUIT \\
\hline $\begin{array}{c}\text { Subset size/ Strain window } \\
\text { size }\end{array}$ & $41 / 9$ & $41 / 11$ & $31 / 13$ & $31 / 11$ \\
$\begin{array}{c}\text { Total error before DIC } \\
\text { parameter optimization } \\
\text { Total error after DIC } \\
\text { parameter optimization }\end{array}$ & 0.0339 & 0.0254 & 0.0487 & 0.0251 \\
\hline
\end{tabular}

Table 7 - Optimization results for the DIC parameters for the four tests

Other DIC parameters that have not been studied so far come into play however. Since the SOAHT specimen exhibits the best identification result after the first two steps, the effects of different step sizes and shape functions were further analysed on this configuration. In the results plotted in Figure 16, the step size was fixed at $50 \%$ of the subset size and the affine shape function was used. A larger overlap of $80 \%$ subset size was simulated and the results are given in Figure 17(a). Consistently with the results provided in [24], the identification error is lower than that for the 50\% overlap.

Then the quadratic shape function was applied with all the other parameters unchanged. In [31], it was concluded that homogeneous displacement fields are better described with an affine subset shape function, whereas a quadratic transformation yields the highest accuracy for the heterogeneous regions. It is shown in Figure 17(b) that the total error is lower for all subset sizes when using quadratic shape functions as opposed to affine ones, confirming the statement from [31]. Shape functions and step size are very rarely considered in DIC literature. The present study underlines their importance and the fact that they should be part of the test optimization process.

The optimal DIC parameters for the SOAHT specimen are listed in Table 8. It shows the DIC parameter optimization has reduced the error from 0.0254 to 0.0173 .

Figure $17-C_{3}$ for the SOAHT specimen: (a) with step as $80 \%$ overlap of the subset, affine shape function; (b) with step as $80 \%$ overlap of the subset, quadratic shape function

\begin{tabular}{ccccc}
\hline Test type & Subset & Step & Strain window & Shape function \\
\hline SOAHT & 41 & 8 & 11 & Quadratic \\
\hline$C_{3}$ & After dimension and off-axis angle optimization: 0.0254 \\
& After subset and strain window optimization: 0.0211 & \\
& After step and shape function optimization:0.0173 & \\
\hline
\end{tabular}

Table 8 - Optimized DIC processing parameters and $C_{3}$ at each optimization step of SOAHT 


\subsection{Optimization of the number of virtual elements}

The influence of different virtual mesh sizes on the identification result is studied in this section. The optimal SOAHT configuration obtained in the previous section is used here. The virtual mesh was $N \times$ $N$ and $N$ was varied from 3 to 11 . Error functions $C_{1}, C_{2}$ and $C_{3}$ are plotted in Figure 19. It can be seen that the systematic error is large when the mesh size is too small. And extra noise is introduced when $N$ is larger than 8 . The random error remains stable between mesh size 5 and 8 and then worsens with the increase of $N$. The total error is at its minimum when $N=5$.

Figure $17-C_{1}, C_{2}$ and $C_{3}$ error functions for the optimal SOAHT configuration with different virtual mesh sizes

\section{Conclusion}

An optimization procedure to compare several potential candidates for the simultaneous identification of the four orthotropic stiffness components of a carbon/epoxy composite has been presented in this paper. A simulator was used to numerically simulate the complete identification chain (from images to inverse computation) for the four test candidates (SOAT, SOAHT, OABD and OAUIT). DIC and VFM were applied to respectively produce the strain fields and identify the stiffness parameters. Error functions were used to evaluate the different test configurations. A two-step procedure was introduced. The first step was to fix the DIC parameters and find the optimal design variables for each test configuration. Then the DIC parameters of each optimized test configuration were analysed to try and reduce the total error further.

The following conclusions can be made:

- After two steps optimization, the SOAHT specimen showed the best identification results. It was retained as the best potential candidate for a new design.

- The effect of some significant DIC parameters (subset size, strain window size, step size and shape function) on the identification results for the SOAHT specimen has been studied. The results indicate that increasing the subset size and the strain window size exerted opposite effects on the systematic and random errors. It is important to balance out the two errors when choosing the optimal DIC processing parameters. The increase of the DIC sampling through a small step size can reduce the total error. And higher order shape functions (quadratic here) can also improve the identification results. This suggests that an adaptive 
approach as the one proposed in [35] and relying on p-adaptive refinement could be of particular interest. This will need to be studied in the future.

This work is a step towards a new standard test based on full-field measurement and inverse identification to obtain composite stiffness components from a single test. The idea has been out for quite a while but the different tools (DIC and VFM) have now reached enough maturity to approach the final design stages. The simulator used here is a powerful tool to address the problem of test design and uncertainty quantification. However, some important steps are still missing.

- When calculating the random part of the identification error, only the noise caused by the sensor of the camera is taken into account. The error arising from the load measurement has been neglected here as this is generally much lower than the errors arising from the imagedbased strain measurements when the load cell is of appropriate capacity compared to the measured load. Other sources of error such as camera misalignment, lens distortion, nonuniform lighting are not considered in this paper and will be included in further studies. In a recent study [38], it was shown for instance that the illumination provides an additional identification error.

- It is clear that all the design parameters (test geometry and loading, imaging hardware, DIC and VFM parameters) are intricately involved and the simple step-wise approach used here is a significant simplification. The next step is now to address the complete optimization problem. Since this mixes continuous and discrete design variables, specialized optimization tools will have to be used. This will be addressed in the near future.

- The other important outstanding issue is the experimental validation. This will be tested soon. Initial results on orthotropic foam characterization [26] has confirmed the relevance of the simulator with experimental results matching expectations in terms of systematic and random errors.

- Finally, the optimal configuration will need to be drafted into a detailed test procedure to be transferred to the composites testing community. This needs to include some difficult issues like the control of the speckle pattern for instance. When stabilized, this procedure may be a good candidate for a future ISO standard.

\section{Acknowledgements}


The authors gratefully acknowledge the financial support of the China Scholarship Council (CSC). Professor Pierron is thankful for the support from the Royal Society and the Wolfson Foundation through a Royal Society Wolfson Research Merit Award.

\section{References}

[1] ASTM, 2014, "D3039 Standard test method for tensile properties of fiber-resin composites."

[2] ASTM, 2013, "D3518 Standard practice for in-plane shear stress-strain response of unidirectional reinforced plastics."

[3] ASTM, 2012, "D5379 / D5379M Standard Test Method for Shear Properties of Composite Materials by the V-Notched Beam Method."

[4] ASTM, 2015, "D4255 Standard guide for testing in-plane shear properties of composites laminates."

[5] Pierron, F., and Vautrin, A., Accurate comparative determination of the in-plane shear modulus of T300/914 by the iosipescu and $45^{\circ}$ off-axis tests. Composites Science and Technology 1994;52(1):6172.

[6] Sun, C. T., and Chung, I., An oblique end-tab design for testing off-axis composite specimens. Composites 1993;24:619-623.

[7] Pierron, F., Saint-Venant effects in the Iosipescu specimen, Journal of Composite Materials 1998;32(22):1986-2015.

[8] Broughton, W., An overview of through-thickness test methods for polymer matrix composites. National Physical Laboratory 1994.

[9] Sutton, M. A., Orteu, J. J., and Schreier, H. W., Image Correlation for Shape, Motion and Deformation Measurements: Basic Concepts, Theory and Applications. Image Correlation for Shape, Motion and Deformation Measurements: Basic Concepts, Theory and Applications 2009;1-321.

[10] Grédiac, M., and Vautrin, A., A new method for determination of bending rigidities of thin anisotropic plates. Journal of Applied Mechanics-Transactions of the Asme 1990;57(4): 964-968.

[11] Gras, R., Leclerc, H., Hild, F., Roux, S., and Schneider, J., Identification of a set of macroscopic elastic parameters in a 3D woven composite: Uncertainty analysis and regularization. International Journal of Solids and Structures 2015;55(0):2-16. 
[12] Pierron, F., Vert, G., Burguete, R., Avril, S., Rotinat, R., and Wisnom, M. R., Identification of the orthotropic elastic stiffnesses of composites with the virtual fields method: sensitivity study and experimental validation. Strain 2007;43(3):250-259.

[13] Gogu, C., Yin, W., Haftka, R., Ifju, P., Molimard, J., Le Riche, R., and Vautrin, A., Bayesian Identification of Elastic Constants in Multi-Directional Laminate from Moiré Interferometry Displacement Fields. Experimental Mechanics 2013;53(4):635-648.

[14] Grédiac, M., Pierron, F., and Surrel, Y., Novel procedure for complete in-plane composite characterization using a single T-shaped specimen. Experimental Mechanics 1999;39(2):142-149.

[15] Avril, S., Bonnet, M., Bretelle, A.-S., Grédiac, M., Hild, F., Ienny, P., Latourte, F., Lemosse, D., Pagano, S., Pagnacco, E., and Pierron, F., Overview of identification methods of mechanical parameters based on full-field measurements. Experimental Mechanics 2008; 48(4):381-402.

[16] Pierron, F., and Grédiac, M., The virtual fields method. Extracting constitutive mechanical parameters from full-field deformation measurements. Springer New-York 2012.

[17] Avril, S., and Pierron, F., General framework for the identification of elastic constitutive parameters from full-field measurements. International Journal of Solids and Structures 2007;44(14-15): 4978-5002. [18] Moulart, R., Avril, S., and Pierron, F., Identification of the through-thickness rigidities of a thick laminated composite tube. Composites Part A: Applied Science and Manufacturing 2006;37(2):326-336. [19] Wang, P., Pierron, F., and Thomsen, O. T., Identification of material parameters of PVC foams using Digital Image Correlation and the Virtual Fields Method. Experimental Mechanics 2013;53(6):10011015.

[20] Chalal, H., Avril, S., Pierron, F., and Meraghni, F., Experimental identification of a nonlinear model for composites using the grid technique coupled to the virtual fields method. Composites Part A: Applied Science and Manufacturing 2006;37(2):315-325.

[21] Surrel, Y., Fournier, N., Grédiac, M., and Paris, P. A., Phase-stepped deflectometry applied to shape measurement of bent plates. Experimental Mechanics 1999;39(1):66-70.

[22] Syed-Muhammad, K., Toussaint, E., Grédiac, M., Avril, S., and Kim, J. H., Characterization of composite plates using the virtual fields method with optimized loading conditions. Composite Structures 2008;85(1):70-82.

[23] Avril, S., Grédiac, M., and Pierron, F., Sensitivity of the virtual fields method to noisy data. Computational Mechanics 2004;34(6):439-452. 
[24] Rossi, M., Lava, P., Pierron, F., Debruyne, D., and Sasso, M., Effect of DIC spatial resolution, noise and interpolation error on identification results with the VFM. Strain 2015; 51(3):206-222.

[25] Rossi, M., and Pierron, F., On the use of simulated experiments in designing tests for material characterization from full-field measurements. International Journal of Solids and Structures 2012;49(34):420-435.

[26] Wang, P., Pierron, F., Thomsen, O. T., Rossi, M., and Lava, P., Uncertainty quantification in VFM identification. Proc. Conference Proceedings of the Society for Experimental Mechanics Series. 137-142. [27] Markides, C. F., Pazis, D. N., Kourkoulis, S. K., Closed full-field solutions for stresses and displacements in the Brazilian disk under distributed radial load. International Journal of Rock Mechanics and Mining Sciences 2010; 47(2):227-237.

[28] Pierron, F., and Grédiac, M., Identification of the through-thickness moduli of thick composites from whole-field measurements using the Iosipescu fixture: theory and simulations. Composites Part A Applied Science and Manufacturing 2000;31(4):309-318.

[29] Chalal, H., Meraghni, F., Pierron, F., and Grédiac, M., Direct identification of the damage behaviour of composite materials using the virtual fields method. Composites Part A: Applied Science and Manufacturing 2004;35(7-8):841-848.

[30] Pierron, F., New Iosipescu fixture for the measurement of the in-plane shear modulus of laminated composites: design and experimental procedure, No. 940125, École Nationale Supérieure des Mines de Saint-Étienne 1994.

[31] Lava, P., Cooreman, S., Coppieters, S., De Strycker, M., and Debruyne, D., Assessment of measuring errors in DIC using deformation fields generated by plastic FEA. Optics and Lasers in Engineering 2009;47(7-8):747-753.

[32] Grediac, M., and Sur, F., 50th Anniversary Article: Effect of Sensor Noise on the Resolution and Spatial Resolution of Displacement and Strain Maps Estimated with the Grid Method. Strain 2014;50(1):1-27.

[33] Badaloni, M., Rossi, M., Chiappini, G., Lava, P., and Debruyne, D., Impact of experimental uncertainties on the identification of mechanical material properties using DIC. Experimental Mechanics 2015;1-16.

[34] Wang, P., Pierron, F., Rossi, M., et al., Optimised Experimental Characterisation of Polymeric Foam Material Using DIC and the Virtual Fields Method. Strain 2016; 52(1):59-79. 
[35] Wittevrongel, L., Lava, P., Lomov, S. V., and Debruyne, D., A Self Adaptive Global Digital Image Correlation Algorithm. Experimental Mechanics 2015;55(2):361-378.

[36] Xavier, J., Avril, S., Pierron, F., and Morais, J., Novel experimental approach for longitudinal-radial stiffness characterisation of clear wood by a single test. Holzforschung 2007;61(5), 573-581.

[37] Xavier, J., Avril, S., Pierron, F., and Morais, J., Variation of transverse and shear stiffness properties of wood in a tree. Composites Part A Applied Science and Manufacturing 2009;40(12), 1953-1960.

[38] Badaloni, M., Rossi, M., Chiappini, G., Lava, P., and Debruyne, D., Impact of experimental uncertainties on the identification of mechanical material properties using DIC. Experimental Mechanics 2015;55(8), 1411-1426.

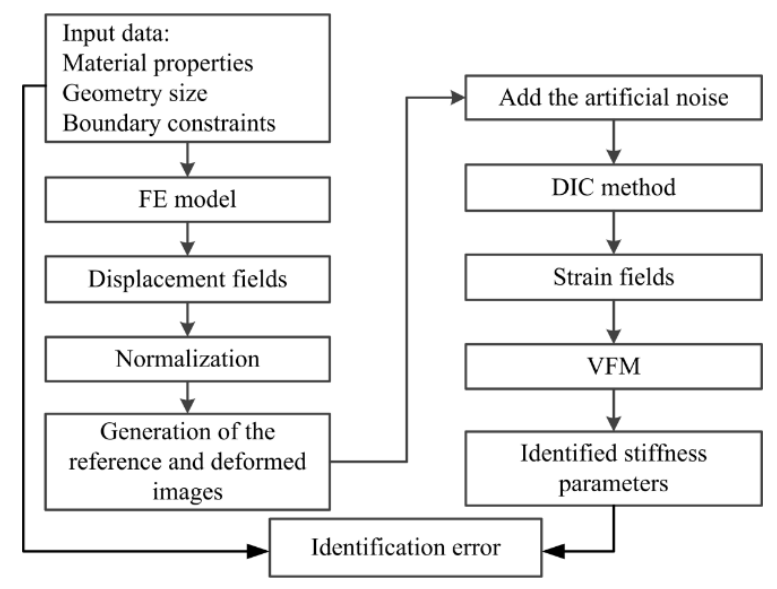

Figure 1 - Flow chart of the identification simulator

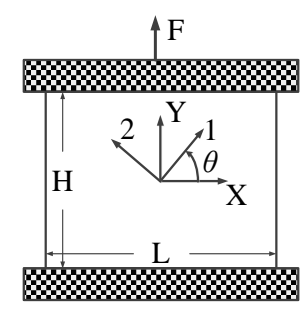

(a)

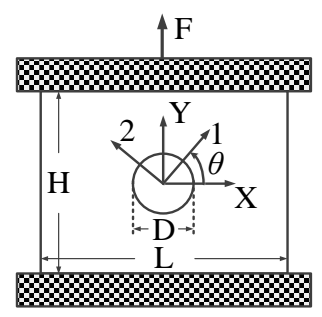

(b) 


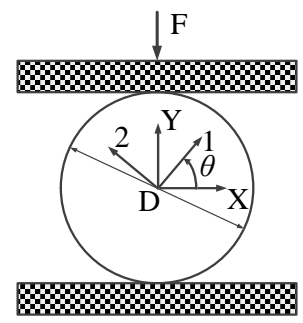

(c)

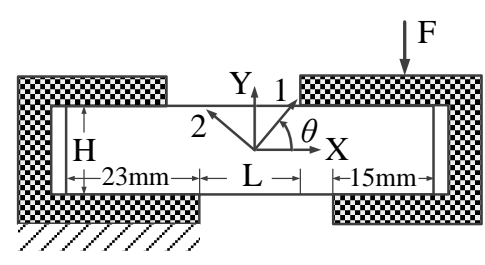

(d)

Figure 2 - FE models of the four types of tests: (a) Short off-axis tensile test (SOAT); (b) Short offaxis open-hole tensile test (SOAHT); (c) Off-axis Brazilian disc (OABD); (d) Off-axis unnotched Iosipescu test (OAUIT)

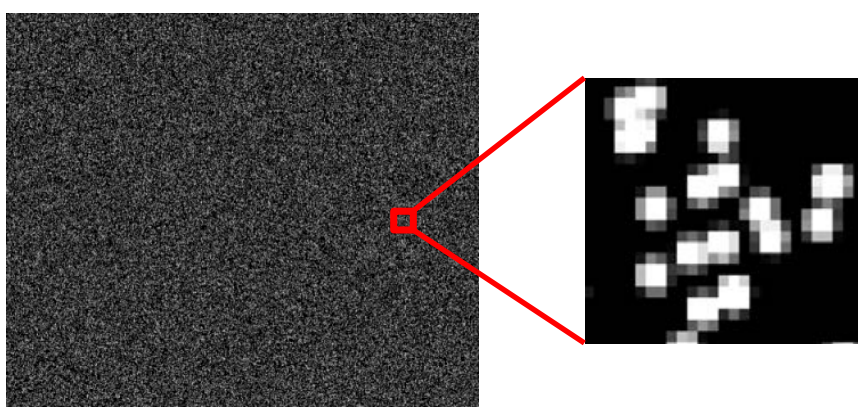

Figure 3 - Reference speckle pattern, with a detail

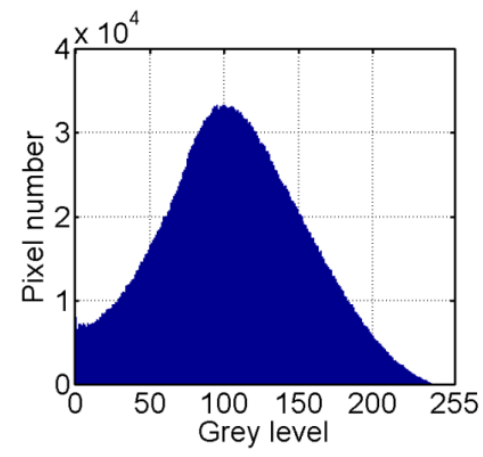

Figure 4 - Grey histogram of the reference image 


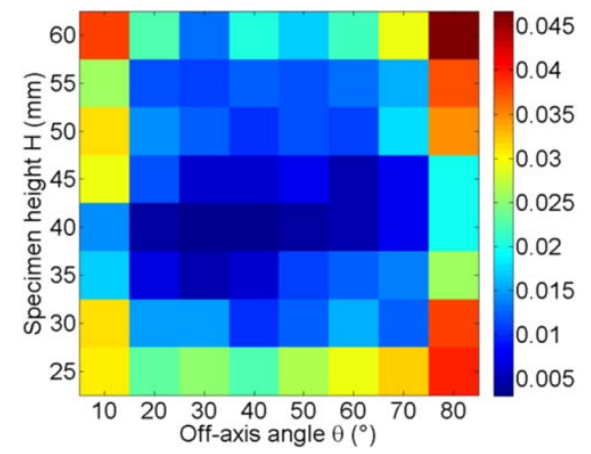

Figure 5 - $C_{1}$ error function for SOAT (systematic error)
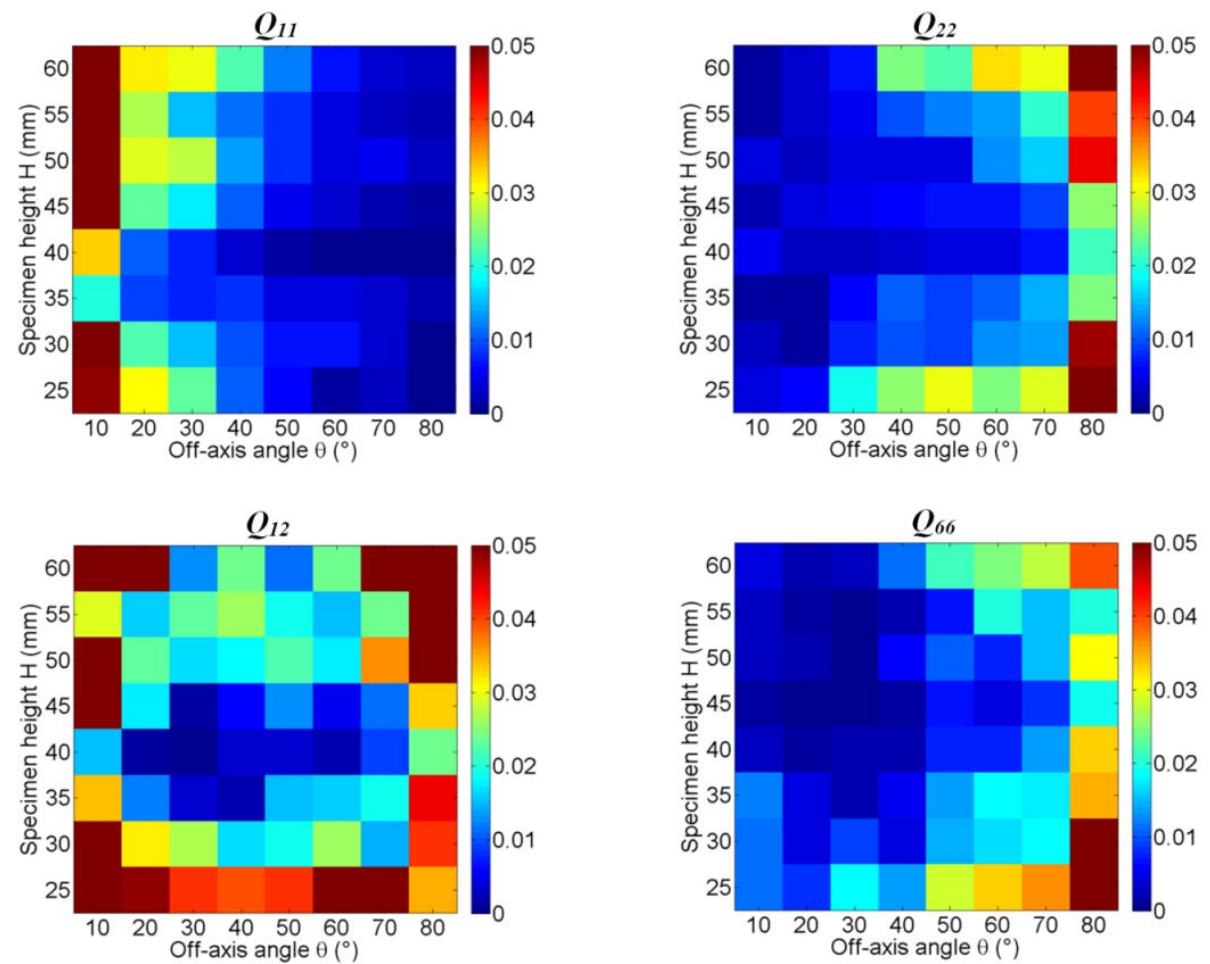

Figure 6 - Systematic error contribution to $C_{1}$ of each identified stiffness parameter for the SOAT configuration 

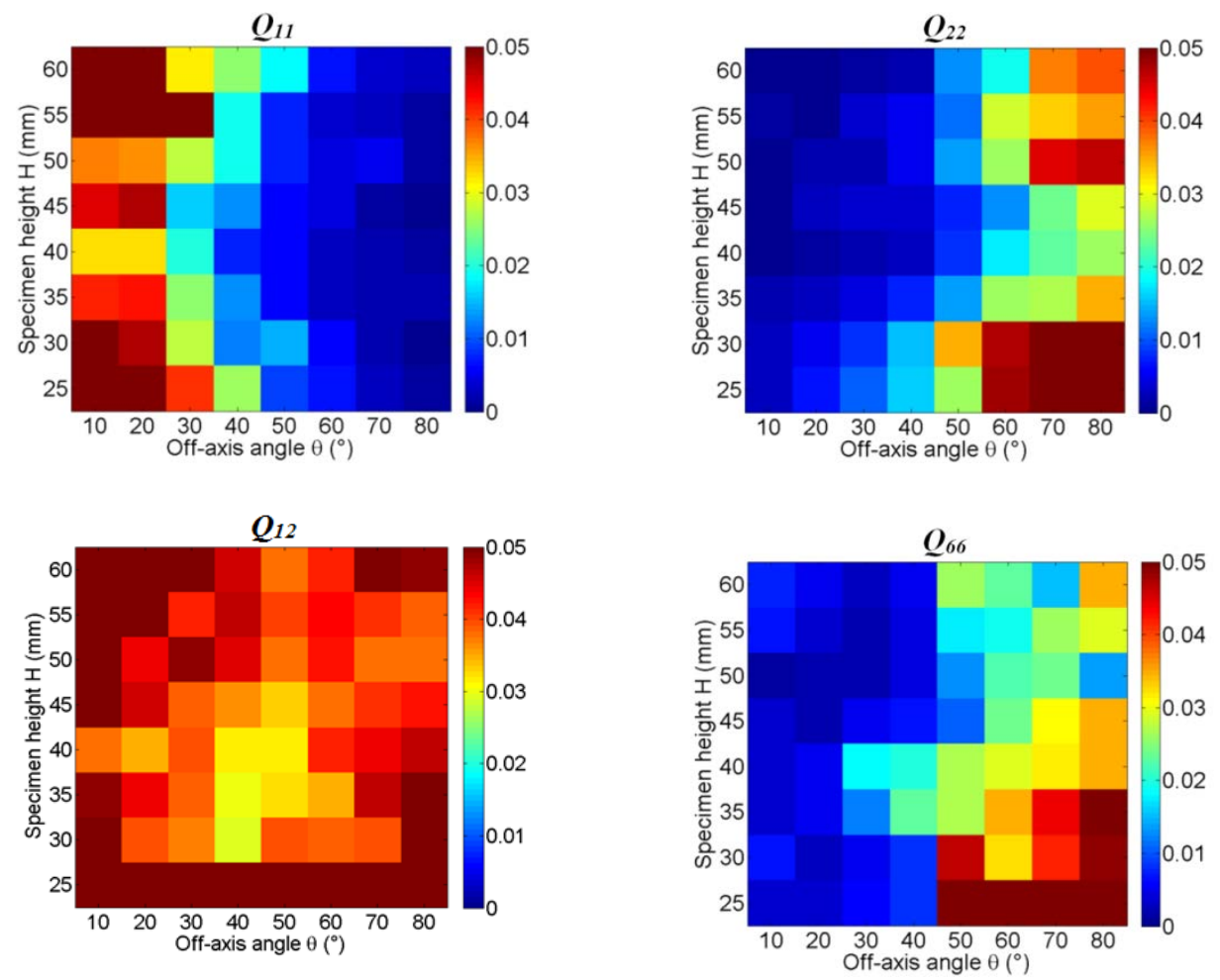

Figure 7 - Coefficients of variation (error function $C_{2}$ ) of each identified stiffness parameter for the SOAT configuration

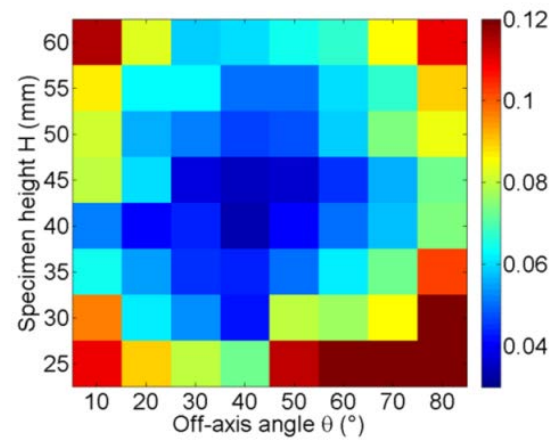

Figure 8 - Total error function $C_{3}$ for the SOAT configuration
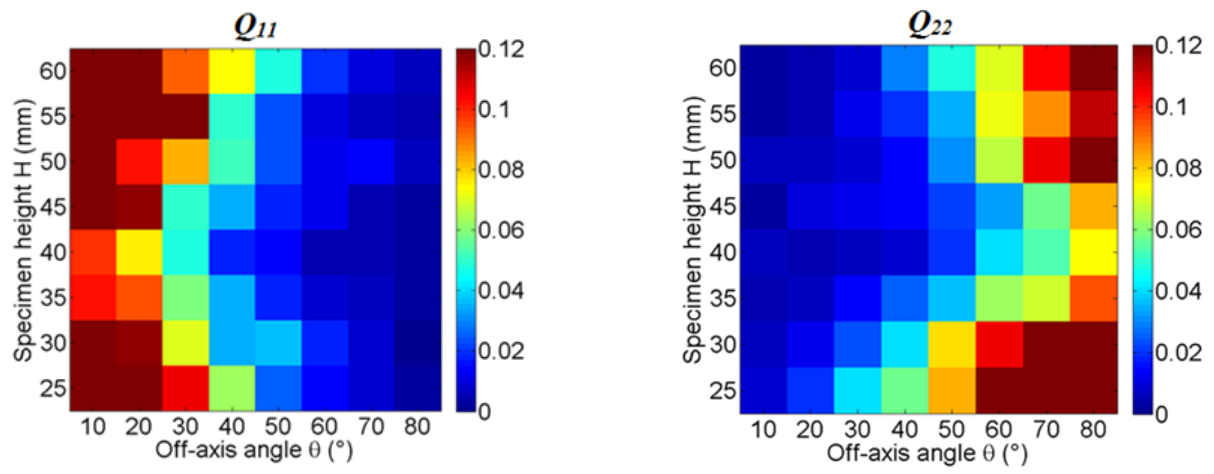

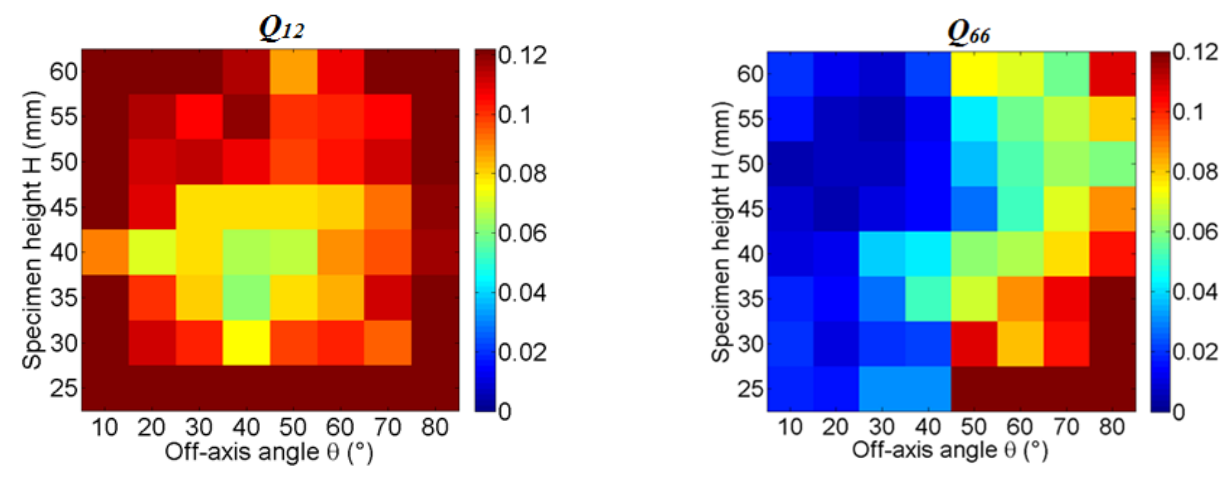

Figure 9 - Total error function $C_{3}$ of each identified stiffness parameter for the SOAT configuration
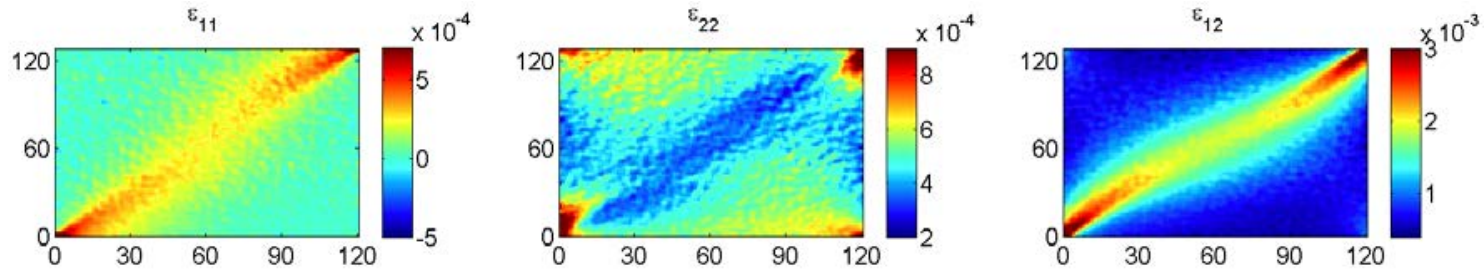

(a)
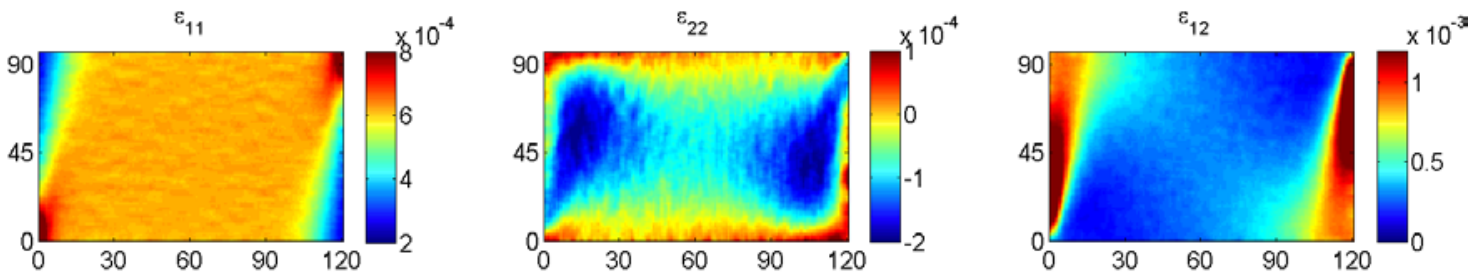

(b)

Figure 10 - Simulated DIC strain maps (with noise) for (a) the optimal SOAT configuration (40 mm, $\left.40^{\circ}\right)$ and (b) a bad one $\left(30 \mathrm{~mm}, 80^{\circ}\right)$
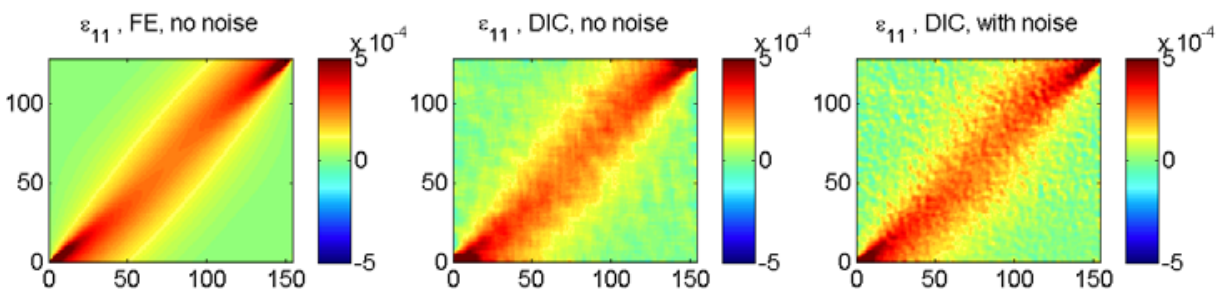

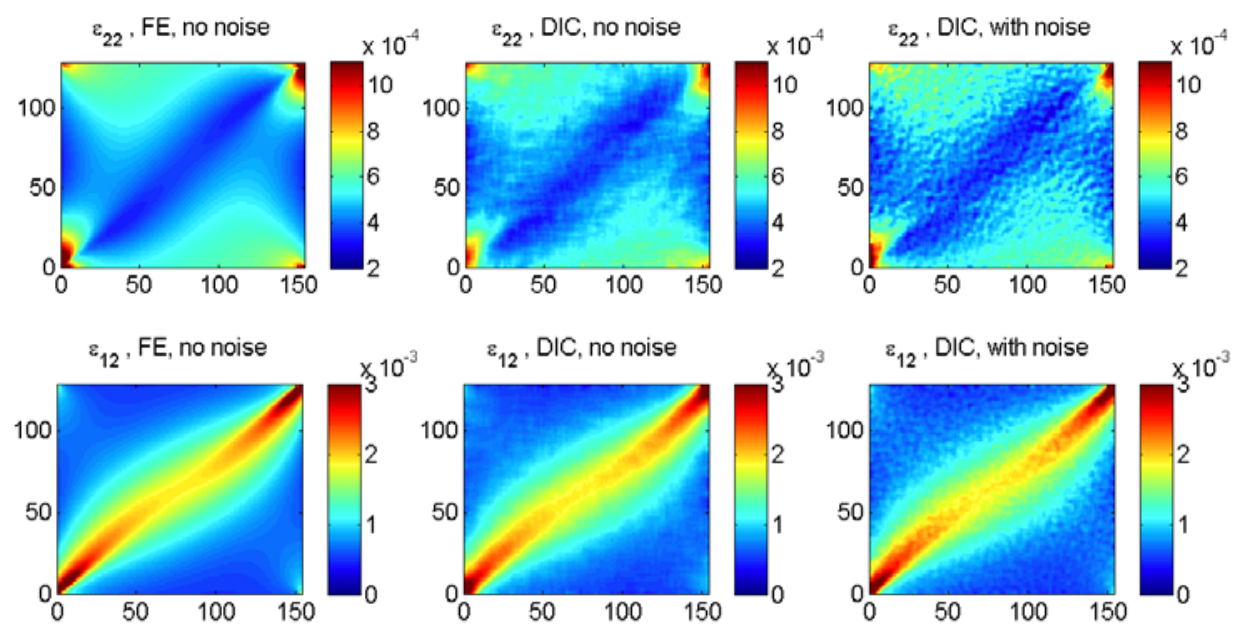

Figure 11 - Strain fields for the optimal SOAT configuration $\left(40 \mathrm{~mm}, 40^{\circ}\right)$, obtained from the FE model, the simulated DIC without and with noise.

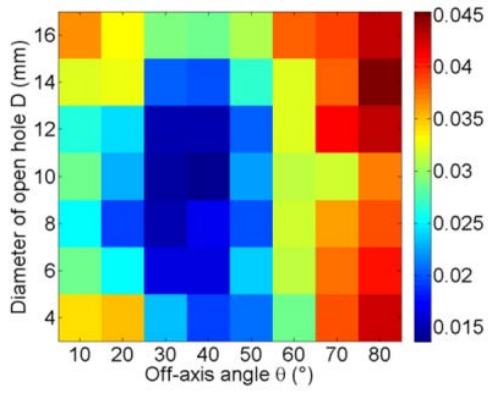

(a)

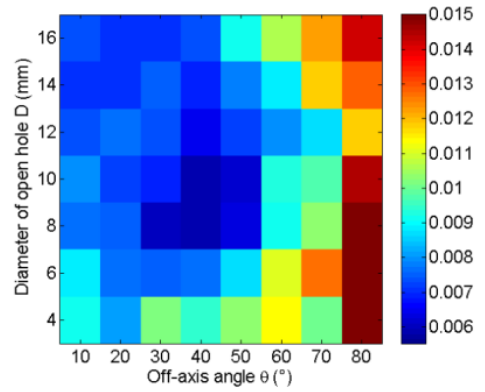

(b)

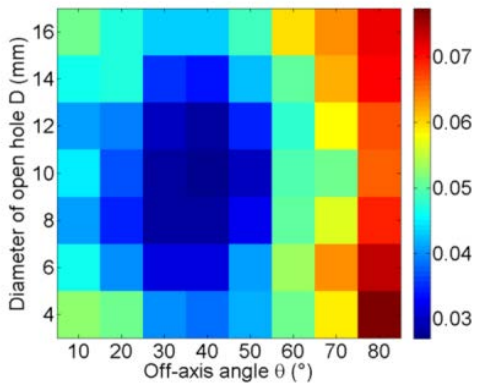

(c)

Figure 12 - (a) $C_{1}$ (bias), (b) $C_{2}$ (random) and (c) $C_{3}$ (total) error funtions for the SOAHT configuration

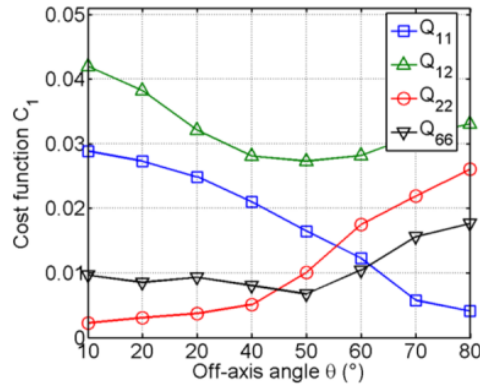

(a)

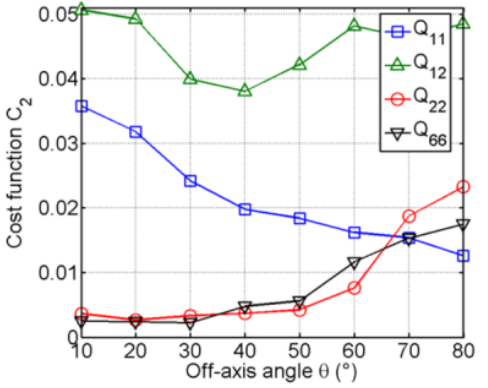

(b)

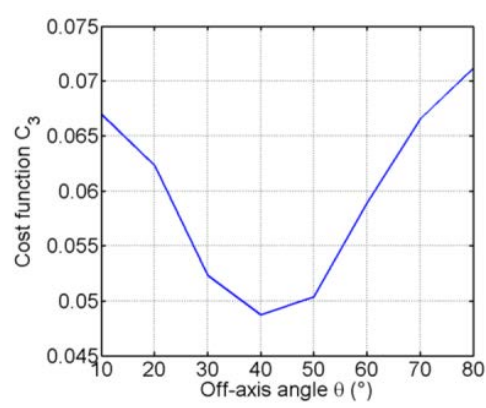

(c)

Figure 13 - (a) Individual $C_{1}$ (bias), (b) Individual $C_{2}$ (random) and (c) global $C_{3}$ (total) error funtions for the OABD configuration 
(b)
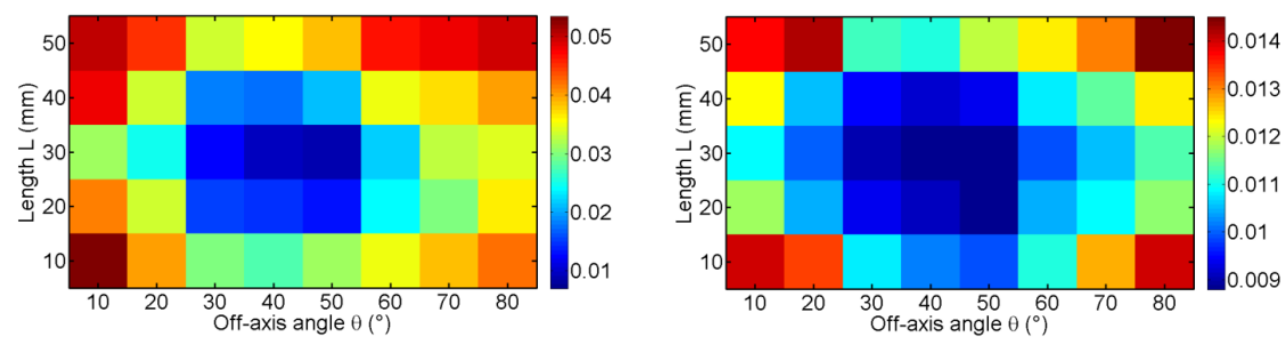

(a)

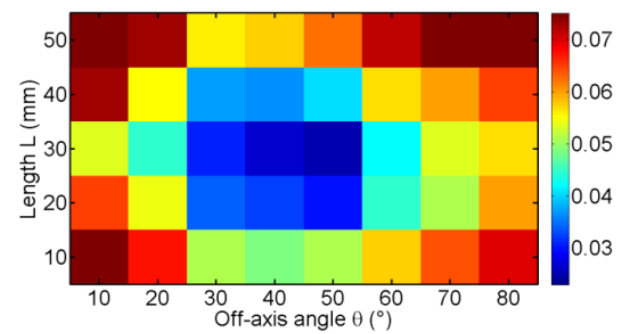

(c)

Figure 14 - (a) $C_{1}$ (bias), (b) $C_{2}$ (random) and (c) $C_{3}$ (total) error funtions for the OAUIT configuration

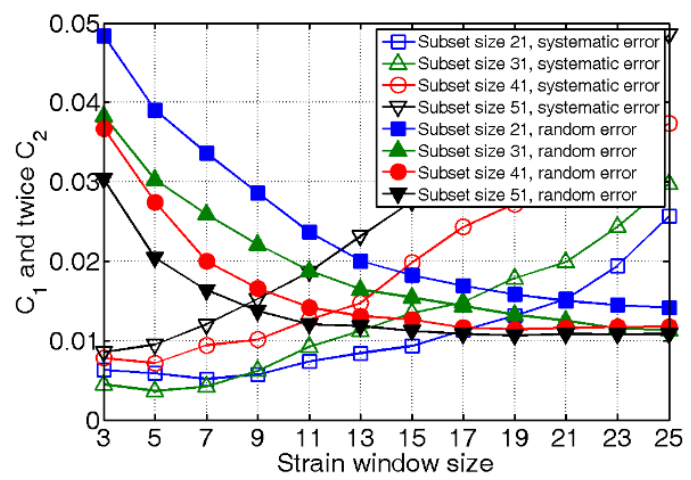

Figure 15 - C1 and twice C2 for the SOAT specimen, with different subset sizes and strain window sizes

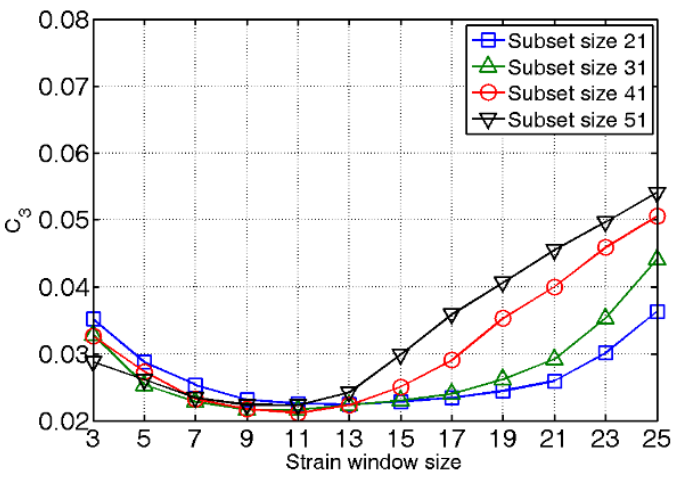

(a)

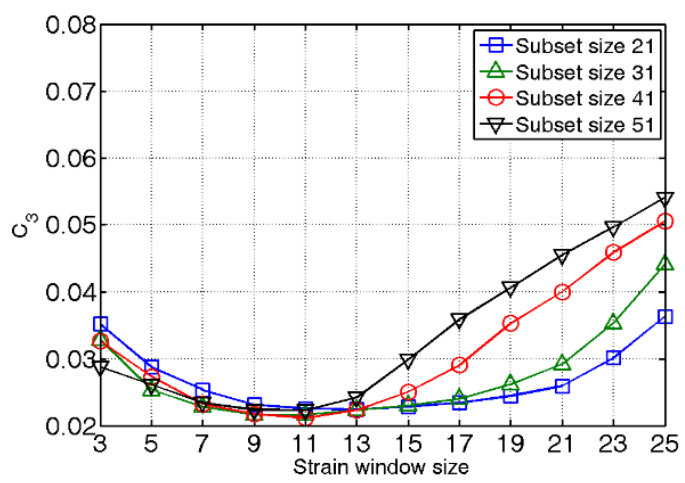

(b) 


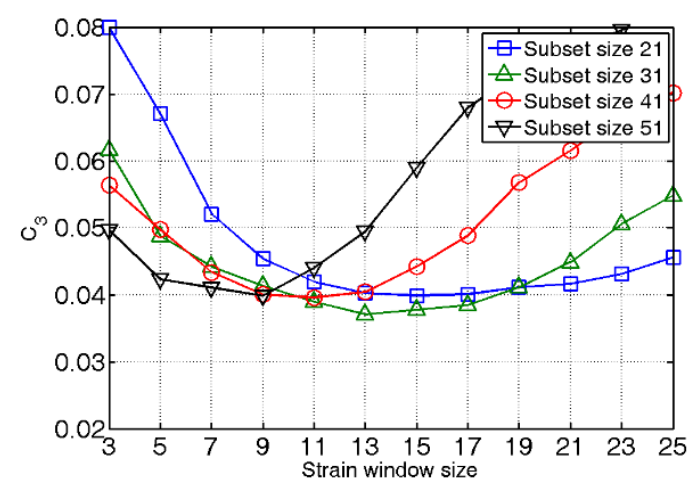

(c)

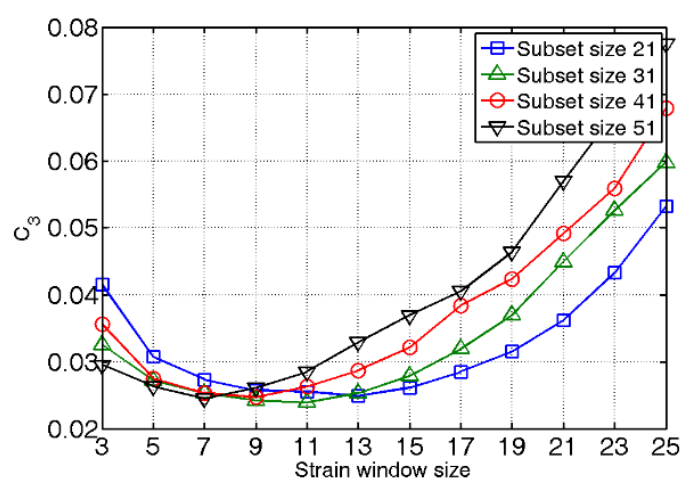

(d)

Figure $16-C_{3}$ of (a) SOAT; (b) SOAHT; (c) OABD; (d) OAUIT, with different subset sizes and strain window sizes

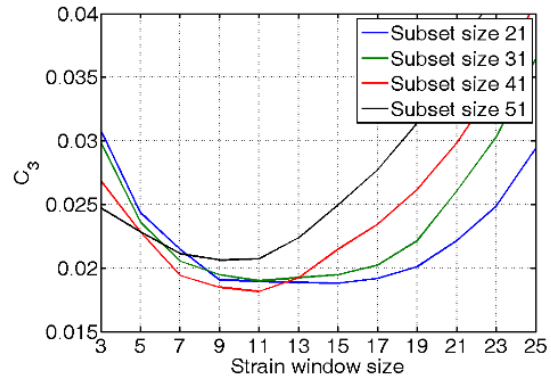

(a)

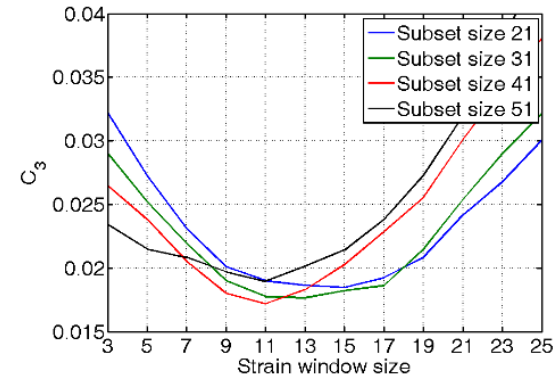

(b)

Figure 17- $C_{3}$ for the SOAHT specimen: (a) with step as $80 \%$ overlap of the subset, affine shape function; (b) with step as $80 \%$ overlap of the subset, quadratic shape function 